OPEN ACCESS

Edited by:

Maarten M. G. van den

Hoogenhof,

Heidelberg University Hospital,

Germany

Reviewed by:

Monika Gladka,

Hubrecht Institute (KNAW),

Netherlands

Li Zhang,

Xinhua Hospital, School of Medicine,

Shanghai Jiao Tong University, China

*Correspondence:

Kexiang Liu

kxliu64@hotmail.com

Weitie Wang

iloveyuiyui@163.com

Specialty section:

This article was submitted to

RNA,

a section of the journal

Frontiers in Genetics

Received: 15 June 2020 Accepted: 30 November 2020

Published: 12 January 2021

Citation:

Wang W, Wang Y, Piao H, Li B, Zhu Z, Li D, Wang T and Liu K (2021)

Bioinformatics Analysis Reveals

MicroRNA-193a-3p Regulates ACTG2 to Control Phenotype Switch

in Human Vascular Smooth Muscle

Cells. Front. Genet. 11:572707.

doi: 10.3389/fgene.2020.572707

\section{Bioinformatics Analysis Reveals MicroRNA-193a-3p Regulates ACTG2 to Control Phenotype Switch in Human Vascular Smooth Muscle Cells}

\author{
Weitie Wang*, Yong Wang, Hulin Piao, Bo Li, Zhicheng Zhu, Dan Li, Tiance Wang and \\ Kexiang Liu*
}

Department of Cardiovascular Surgery of the Second Hospital of Jilin University, The Second Hospital of Jilin University, Changchun, China

Aortic dissection (AD) is among the most fatal cardiovascular diseases. However, the pathogenesis of $A D$ remains poorly understood. This study aims to integrate the microRNAs (miRNA) and mRNA profiles and use bioinformatics analyses with techniques in molecular biology to delineate the potential mechanisms involved in the development of $A D$. We used the human miRNA and mRNA microarray datasets GSE98770, GSE52093, and GEO2R, Venn diagram analysis, gene ontology, and protein-protein interaction networks to identify target miRNAs and mRNAs involved in AD. RNA interference, western blotting, and luciferase reporter assays were performed to validate the candidate miRNAs and mRNAs in AD tissues and human vascular smooth muscle cells (VSMCs). Furthermore, we studied vascular smooth muscle contraction in AD. In silico analyses revealed that miR-193a-3p and ACTG2 were key players in the pathogenesis of $A D$. miR-193a-3p was upregulated in the $A D$ tissues. We also found that biomarkers for the contractile phenotype in VSMCs were downregulated in AD tissues. Overexpression and depletion of miR-193a-3p enhanced and suppressed VSMC proliferation and migration, respectively. Dual luciferase reporter assays confirmed that ACTG2 was a target of miR-193a-3p. ACTG2 was also downregulated in human AD tissues and VMSCs overexpressing miR-193a-3p. Taken together, miR-193a-3p may be a novel regulator of phenotypic switching in VSMCs and the miR-193a-3p/ACTG2 axis may serve as a promising diagnostic biomarker and therapeutic candidate for $A D$.

\section{Keywords: mRNA, miRNA, vascular disease, aortic dissection, cardiovascular}

\footnotetext{
Abbreviations: AD, aortic dissection; VSMCs, vascular smooth muscle cells; miRNAs, microRNAs; GEO, gene expression omnibus; GO, gene ontology; KEGG, Kyoto Encyclopedia of Genes and Genomes; DEGs, the differentially expressed genes; CTA, computed tomography angiography; PPI, protein-protein interaction; qRT-PCR, quantitative reverse transcriptionPCR; CCK, Cell Counting Kit; FDR, false discovery rate; DEMs, differential expression profile of miRNAs; CAD, coronary heart disease; CC, cellular component; BP, biological processes; MF, molecular function.
} 


\section{INTRODUCTION}

Type A aortic dissection (AD) is a fatal cardiovascular disease associated with high morbidity and mortality, and requires a complex treatment regimen (Elsayed et al., 2017; Silaschi et al., 2017). Vascular smooth muscle cells (VSMCs) are involved in vascular function and have been implicated in the pathogenesis of AD (Zhang et al., 2016; Wei et al., 2017). However, the precise mechanisms involved in $\mathrm{AD}$ remain to be fully understood. Therefore, it is important to delineate the roles of VSMCs in AD and phenotypic plasticity. This will help identify new modes of treatment, especially targeted drug therapy. Owing to advances in sequencing technology, differential genes expression between normal and damaged tissues have been widely used to identify candidate pathogenic genes (Wang et al., 2019a). Bioinformatics tools can analyze such high-throughput data, while omitting "junk" data to provide reliable analyses.

Vascular smooth muscle cells phenotypic remodeling is mainly relevant with intima-media thickening which acts important roles during the pathological progression for vascular disease, such as AD (Gomez and Owens, 2012). Although the pathogenesis of $\mathrm{AD}$ remains unclear, VSMCs phenotypic remodeling alternating from contractile to synthetic in response to stimulation are essential in $\mathrm{AD}$, which regulates vascular remodeling. Contractile VSMCs generally demonstrate reduced ability in proliferation and migration, whereas synthetic VSMCs present enhanced viability in proliferation and migration (Alexander and Owens, 2012). Downregulation of differentiation markers such as SMA, SM22, and MYH11 always happen in synthetic VSMCs. Therefore, detection of differentiation markers suggests the VSMCs phenotypic remodeling. Numerous factors including growth factor and cell adhesion molecules promote the phenotypic switch to synthetic VSMCs and enhance cell proliferation and migration (Gomez and Owens, 2012). However, the molecular mechanisms between VSMC phenotypic switch and $\mathrm{AD}$ remain unclear.

MicroRNAs (miRNAs) are non-coding RNAs that function by binding to target mRNAs. miRNAs regulate various cellular functions, including proliferation and migration, and phenotypic switch in VSMCs (Tang et al., 2017; Li et al., 2019). Therefore, it is imperative to understand the importance of miRNAs and their targets in VSMC function in patients with $\mathrm{AD}$.

In this study, we combined bioinformatics analysis with techniques in molecular biology to elucidate key miRNAs and mRNAs involved in AD. GEO2R analysis, target gene prediction, gene ontology (GO) and pathway analysis, Venn diagrams, and protein-protein interaction (PPI) networks were used to assess the roles of miR-193a-3p/ACTG2 as candidates associated with phenotypic switching in VSMCs. Our study presented that in vitro cell culture-based experiments revealed miR-193a3 p/ACTG2 participated in the development of AD.

\section{MATERIALS AND METHODS}

\section{Datasets and Workflow}

Human datasets GSE98770 (GPL14550) and GSE52093 (GPL10558) were obtained from the GEO database. We analyzed
11 samples from GSE98770 (six AD and five normal ascending tissues) and 12 samples from GSE52093 (seven AD and five normal ascending tissues). Figure 1 shows the workflow used in the study.

\section{Analyzing the Differential Expression of RNAs}

GEO2R with $R$ software was used to determine the gene expression profiles of $\mathrm{AD}$ and normal control (NC) samples and identify genes that were differentially expressed. Data from normal and $\mathrm{AD}$ samples were arranged in order before using the R software. $P<0.05$ and $\mid$ Log fold change $\mid \geq 1.0$ were used as the screening thresholds for datasets GSE98770 and GSE52093.

\section{Bioinformatic Analysis}

All differentially expressed genes (DEGs) were analyzed using the DAVID online tool that included GO and Kyoto Encyclopedia of Genes and Genomes (KEGG) functional enrichment. The top pathways associated with the DEGs were further analyzed. We had previously determined the mRNA profiles for six AD and normal tissues each (Wang et al., 2019a) that were used to compare within dataset GSE52093 to identify the DEGs.

\section{Target mRNA Identification and Generation of the PPI Network}

We generated the PPI network using geneMANIA ${ }^{1}$ for the DEGs associated with vascular smooth muscle contraction in dataset GSE52093 and our mRNA profiles. A confidence score $>0.4$ was considered significant.

\section{Prediction of Differentially Expressed miRNAs (DEMs)}

All miRNAs expressed in GSE52093 and the predicted mRNA profiles correlating with vascular smooth muscle contraction were selected to predict the DEMs using TargetScan. ${ }^{2}$

\footnotetext{
${ }^{1}$ https://genemania.org

${ }^{2}$ www.targetscan.org
}

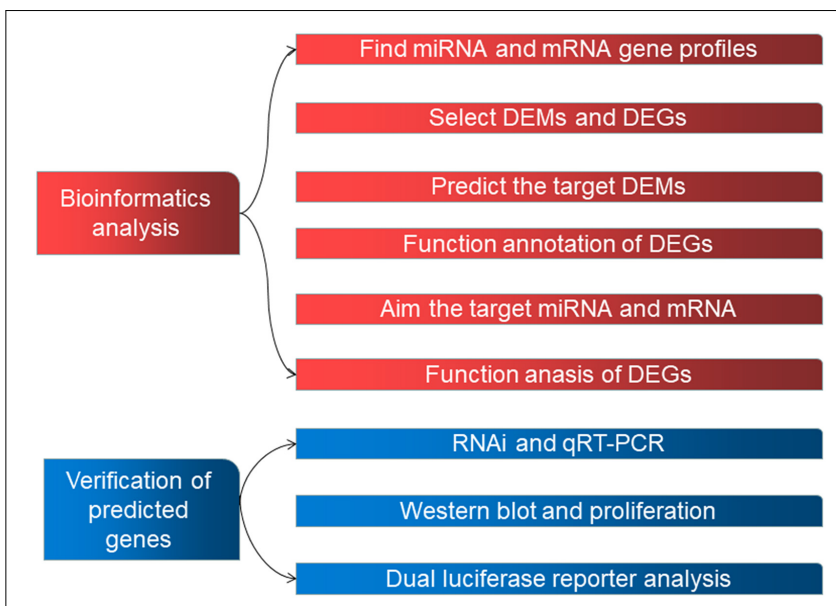

FIGURE 1 | The workflow used in this study. 


\section{Venn Diagram Analysis}

Differentially expressed miRNAs from the GSE98770 dataset and miRNAs predicted in this study were analyzed using Venn diagrams generated by using FunRich (Zhang and Wang, 2019).

\section{Human Aortic Samples}

This study was conducted in accordance with the Declaration of Helsinki and was approved by the Ethical Committee at Jilin University (IRB:2019018). All patients agreed to participate in this study and provided written informed consent. Human ascending aorta specimens were acquired from AD patients $(n=10)$ and ischemic heart disease patients $(n=10)$ during surgery (Table 1). All AD patients were diagnosed using computed tomography angiography and patients with hereditary disease were excluded.

\section{Cell Culture}

Human aortic VSMCs (Lonza, Walkersville, MD, United States) were cultured in smooth muscle culture medium (SMCM) (America Sciencell) supplemented with $2 \%$ fetal bovine serum and $1 \%$ smooth muscle cell growth supplement at $37^{\circ} \mathrm{C}$ in a humidified $5 \% \mathrm{CO}_{2}$ incubator. Cells used in this study were between passage number 5 and 7 . VSMCs were serum starved $(0.5 \%$ fetal bovine serum $)$ for $24 \mathrm{~h}$ and stimulated using PDGF-BB.

\section{Quantitative Reverse Transcription-Polymerase Chain Reaction}

Total RNA was extracted using TRIzol (Invitrogen) according to the protocol provided. After measuring RNA concentration at $260 \mathrm{~nm}$, the total RNA was reverse transcribed to cDNA (Takara Bio Inc., Japan) to analyze the mRNA and miRNA content in samples. Table 2 lists all the primers used in this study. Glyceraldehyde-3-phosphate dehydrogenase (GAPDH) and U6 were used as endogenous controls. We calculated relative gene expression using the $2^{-\Delta \Delta \mathrm{Ct}}$ method.

TABLE 1 | Information of aortic dissection and ischemic heart disease patients.

\begin{tabular}{lcc}
\hline & AD group & control group \\
\cline { 2 - 3 } & (n= 10) & $(\boldsymbol{n}=\mathbf{1 0})$ \\
\hline Age (years old) & $48.3 \pm 7.31$ & $58.22 \pm 5.91$ \\
Male & $6(60.00 \%)$ & $6(60.00 \%)$ \\
Maximal diameter, cm & $5.81 \pm 2.09$ & $2.98 \pm 0.34$ \\
Smoking & $2(20.00 \%)$ & $6(60.00 \%)$ \\
NYHA class III-IV & $1(10.00 \%)$ & $4(20.00 \%)$ \\
Hypertension & $7(70.00 \%)$ & $6(60.00 \%)$ \\
Diabetes mellitus & $0(00.00 \%)$ & $6(60.00 \%)$ \\
Chronic renal dysfunction & 0 & 0 \\
\hline
\end{tabular}

$A D$, aortic dissection; NYHA, New York Heart Association.
TABLE 2 | The primers in this work.

\begin{tabular}{lll}
\hline $\begin{array}{l}\text { Gene } \\
\text { name }\end{array}$ & Forward primer & Reverse primer \\
\hline $\begin{array}{lll}\text { miR- } \\
\text { 193a-3p }\end{array}$ & ATGCTCAAACTGGCCTACAAG & $\begin{array}{l}\text { TATGGTTGTCTGCTCTCT } \\
\text { GTCTC }\end{array}$ \\
ACTG2 & GCGTGTAGCACCTGAAGAG & GAATGGCGACGTACATGGCA \\
SMA & GCGTGGCTATTCCTTCGTA & ATGAAGGATGGCTGGAACAG \\
Calponin & AGCTAAGAGAAGGGCGGAAC & CATCTGCAGGCTGACATTGA \\
SM22a & AACAGCCTGTACCCTGATGG & CGGTAGTGCCCATCATTCT \\
MMP-2 & ACCCATTACACCTACACCAAG & TGTTGCAGATCTCAGGAGTG \\
MMP-9 & CGAACTTGACAGCGACAAG & CACTGAGGAATGATCTAAGCCC \\
MYH11 & TGGAACTTCATCGACTTTGGG & ACAGCTTCTCCACGAAAGAC \\
U6 & GCGCGTCGTGAAGCGTTC & GTGCAGGGTCCGAGGT \\
GAPDH & CGGACCAATACGACCAAATCCG & AGCCACATCGCTCAGACACC
\end{tabular}

miR-193, microRNA-193; ACTG2, actin gamma 2; SMA, smooth muscle - actin; SM22, smooth muscle 22; MYH11, smooth muscle myosin heavy chain.

\section{VSMC Transfection}

The miR-193a-3p mimic and inhibitor were designed by GenePharma (Shanghai, China). VSMCs were transfected with 250 pmol of the mimic or inhibitor and $2 \mu \mathrm{l}$ Transfection Reagent (TransDetect, Beijing, China) for $\sim 5 \mathrm{~h}$. Cellular miRNA expression were analyzed $48 \mathrm{~h}$ after transfection.

\section{Western Blot Analysis}

Total protein content of the cell was separated using sodium dodecyl sulfate-polyacrylamide gel electrophoresis and transferred to nitrocellulose membranes. Blots were blocked using 5\% non-fat milk supplemented with $0.1 \%$ Tween 20 . Subsequently, the blots were incubated with primary antibodies against ACTG2 (1:500, bioss), SM22a (1:500, Proteintech), SMA (1:800, bioss), calponin (1: 2,000, bioss), MYH (1:1,000, Proteintech), MMP-2 (1:500, abcam), and MMP-9 (1:500, abcam) followed by incubation with secondary antibodies. The images were analyzed using ImageJ.

\section{Dual Luciferase Reporter Assay}

Wild or mutant $3^{\prime}$-untranslated region (UTR) of ACTG2 were cloned into pGL6-miR (Beyotime, China). We seeded $2 \times 10^{4}$ HEK293T cells onto six-well plates for $24 \mathrm{~h}$ before transfection. The luciferase kit (Beyotime) was used after transfection (pGL6ACTG2-wt and pGL6-ACTG2-mut) using Lipofectamine 2000 (Invitrogen, Life Technologies). Luciferase activity was measured at $560 \mathrm{~nm}$. Signal from Renilla luciferase was normalized to that from firefly luciferase.

\section{Cell Proliferation Assay}

We seeded $\sim 3 \times 10^{4}$ cells VSMC in each well of a 96well plate for $15-20 \mathrm{~h}$ before transfection. Cells were then transfected with the miR-193a-3p mimic and inhibitor and incubated for $20 \mathrm{~h}$. Subsequently, the cells were incubated with medium containing the Cell Counting Kit (CCK) Solution (TransDetect, Beijing, China) for $4 \mathrm{~h}$ following which we measured absorbance at $450 \mathrm{~nm}$. 


\section{Wound Healing Assay}

We seeded $2.5 \times 10^{5}$ cells into each well of a six-well plate and transfected them with miR-193a-3p. After starving for $48 \mathrm{~h}$, we generated a linear wound using a 200- $\mu$ l tip. Cells were then incubated in medium for 48 and $72 \mathrm{~h}$. Finally, cells that migrated into the wounded area were counted.

\section{Statistical Analysis}

Continuous data are expressed as mean \pm standard deviation. One-way analysis of variance (ANOVA) was performed between-group differences and multiple groups. Comparisons among multiple groups were performed using one-way ANOVAs, followed by post hoc Tukey tests. Relative expression of RT-qPCR was calculated using the $\Delta \Delta \mathrm{Cq}$ method.

\section{RESULTS}

\section{DEGs in AD}

All the raw data were first analyzed using the GEO2R tool. From the GSE98770 dataset, we identified 34 and 47 upregulated and downregulated miRNAs, respectively. From the GSE52093 dataset, we identified 1,409 and 1,235 upregulated and downregulated mRNAs, respectively. The volcano plots showed the DEMs (Figure 2A) and DEGs (Figure 2B) in the $\mathrm{AD}$ and $\mathrm{NC}$ samples.

\section{GO and KEGG Pathway Analysis}

DAVID was used to functionally analyze the DEGs. The downregulated mRNAs correlated with muscle

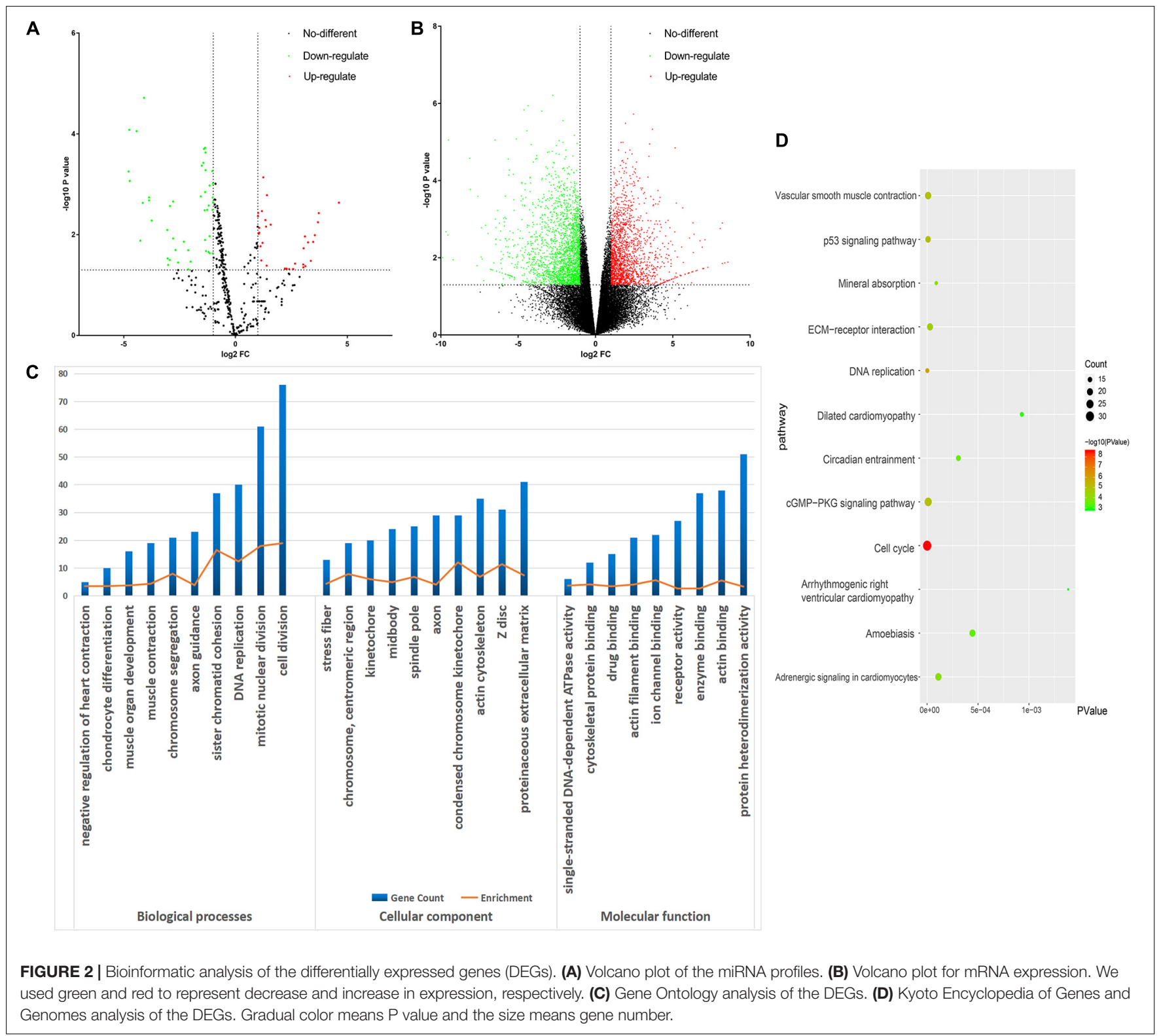


contraction and muscle organ development in $\mathrm{AD}$, suggesting that downregulated genes primarily localized to the aortic media. Upregulated DEGs were involved in cell division and mitotic nuclear division, indicating the importance of cell division in AD (Table 3 and Figure 2C).

Kyoto Encyclopedia of Genes and Genomes analysis revealed that the downregulated and upregulated genes were involved in vascular smooth muscle contraction and cell cycle, respectively (Figure 2D). Thus, GO and KEGG analyses highlighted the importance of vascular smooth muscle contraction in the aortic media during $\mathrm{AD}$.

\section{PPI Network}

We generated a PPI network with genes related to the vascular smooth muscle contraction pathway using GeneMANIA bioinformatic analysis. Results showed that 8 genes (ACTG2 and PPP1R12B predicted by DAVID tool. MYH11, EDNRA, MYL2, ROCK2, ROCK1, and KCNQ1 predicted by GeneMANIA) were involved in muscular system processes (FDR:1.40e-7, nine gene count), muscle contraction (FDR:1.26e-6, eight gene count), and smooth muscle contraction (FDR:1.65e-3, four gene count) (Figure 3A). Then ACTG2 and PPP1R12B were used for PPI network analysis by GeneMANIA, which also indicated changing muscle function was relevant in AD (Figure 3B). Thus, combined with PPI analyses and previous GO and KEGG analyses, ACTG2 and PPP1R12B may be crucial genes involved in AD.

\section{Target Gene Prediction and Validation}

ACTG2 and PPP1R12B were used to predict potential target miRNAs by TargetScan. About 881 predicted miRNAs for ACTG2 and 776 predicted miRNAs for PPP1R12B (score $\geq 80$ ) were collected and compared to 81 candidate miRNAs from GSE98770 to identify DEMs. Venn diagram analysis showed 49 DEMs (Figure 3C) that were common to data from GSE98770 and the miRNAs predicted in this study. A miRNA-mRNA network was constructed using Cytoscape (Figure 3D). miR-193a-3p was upregulated and among the top 3 predicted miRNAs with high score and binding sites for ACTG2. Furthermore, miR-193a-3p is crucial for cell proliferation. Therefore, miR-193a-3p may regulate VSMC proliferation.

TABLE 3 | Enriched analysis.

\begin{tabular}{|c|c|c|c|c|c|}
\hline Expression & Category & Term & Description & Gene count & $P$-value \\
\hline & $\mathrm{BP}$ & mitotic nuclear division & GO:0007067 & 61 & 1.36E-18 \\
\hline & $\mathrm{BP}$ & DNA replication & GO:0006260 & 40 & 4.61E-13 \\
\hline & $\mathrm{BP}$ & chromosome segregation & GO:0007059 & 21 & 1.29E-08 \\
\hline & $\mathrm{CC}$ & condensed chromosome kinetochore & GO:0000777 & 29 & $1.02 \mathrm{E}-12$ \\
\hline & $\mathrm{CC}$ & cytosol & GO:0005829 & 290 & 3.39E-07 \\
\hline & $\mathrm{CC}$ & membrane & GO:0016020 & 204 & 6.67E-07 \\
\hline & MF & protein binding & GO:0005515 & 643 & 2.32E-04 \\
\hline & MF & single-stranded DNA-dependent ATPase activity & GO:0043142 & 6 & 2.46E-04 \\
\hline & MF & drug binding & GO:0008144 & 15 & 4.36E-04 \\
\hline & $\mathrm{BP}$ & axon guidance & GO:0007411 & 23 & 1.69E-04 \\
\hline & $\mathrm{BP}$ & muscle organ development & GO:0007517 & 16 & 2.09E-04 \\
\hline & $\mathrm{BP}$ & chondrocyte differentiation & GO:0002062 & 10 & 3.58E-04 \\
\hline & $\mathrm{BP}$ & negative regulation of heart contraction & GO:0045822 & 5 & 3.67E-04 \\
\hline & $\mathrm{CC}$ & Z disc & GO:0030018 & 31 & 4.67E-12 \\
\hline & $\mathrm{CC}$ & proteinaceous extracellular matrix & GO:0005578 & 41 & 4.59E-08 \\
\hline & $\mathrm{CC}$ & actin cytoskeleton & GO:0015629 & 35 & 1.60E-07 \\
\hline & $\mathrm{CC}$ & plasma membrane & GO:0005886 & 296 & 4.28E-05 \\
\hline & $\mathrm{CC}$ & stress fiber & GO:0001725 & 13 & 5.30E-05 \\
\hline & MF & calcium ion binding & GO:0005509 & 78 & 2.79E-07 \\
\hline & MF & ion channel binding & GO:0044325 & 22 & 2.68E-06 \\
\hline
\end{tabular}




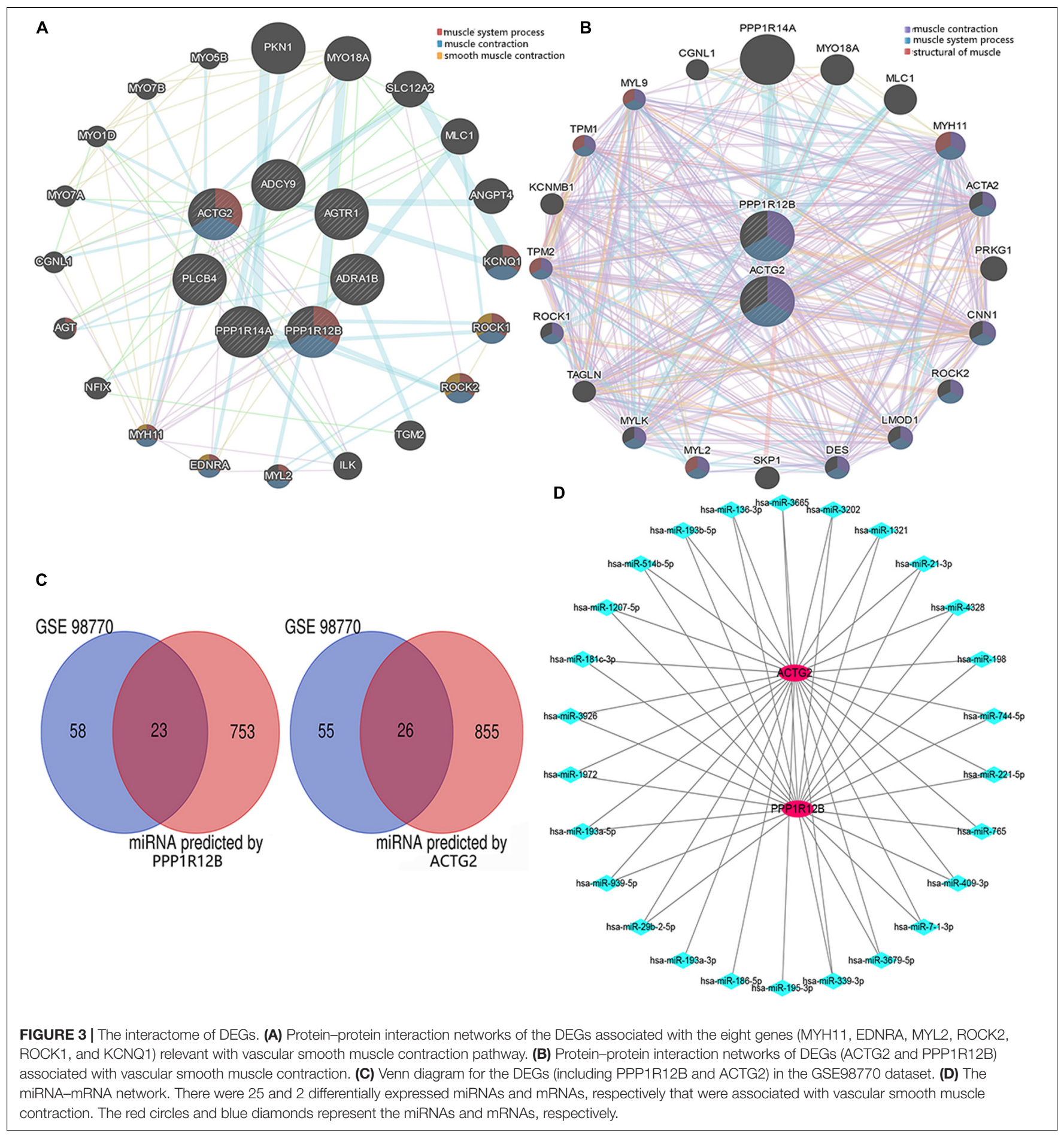

\section{miR-193a-3p Regulates VSMC Phenotypes}

Tissues near the intimal tear isolated from patients with $\mathrm{AD}$ exhibited decreased expression of differentiation biomarkers (SMA, SM22, calponin, and MYH11) and contained highly proliferative VSMCs compared to those in tissues from healthy individuals (Figures $4 \mathbf{A}, \mathbf{B}$ ). At the meantime, miR-193a-3p also increased in the
AD tissues (Figure 4C). These results highlight the involvement of miR-193a-3p in human VSMC function and phenotypic plasticity.

\section{Overexpression of miR-193a-3p in Proliferative VSMCs}

To identify that miR-193a-3p is specifically involved in the regulation of VSMC, quantitative reverse 


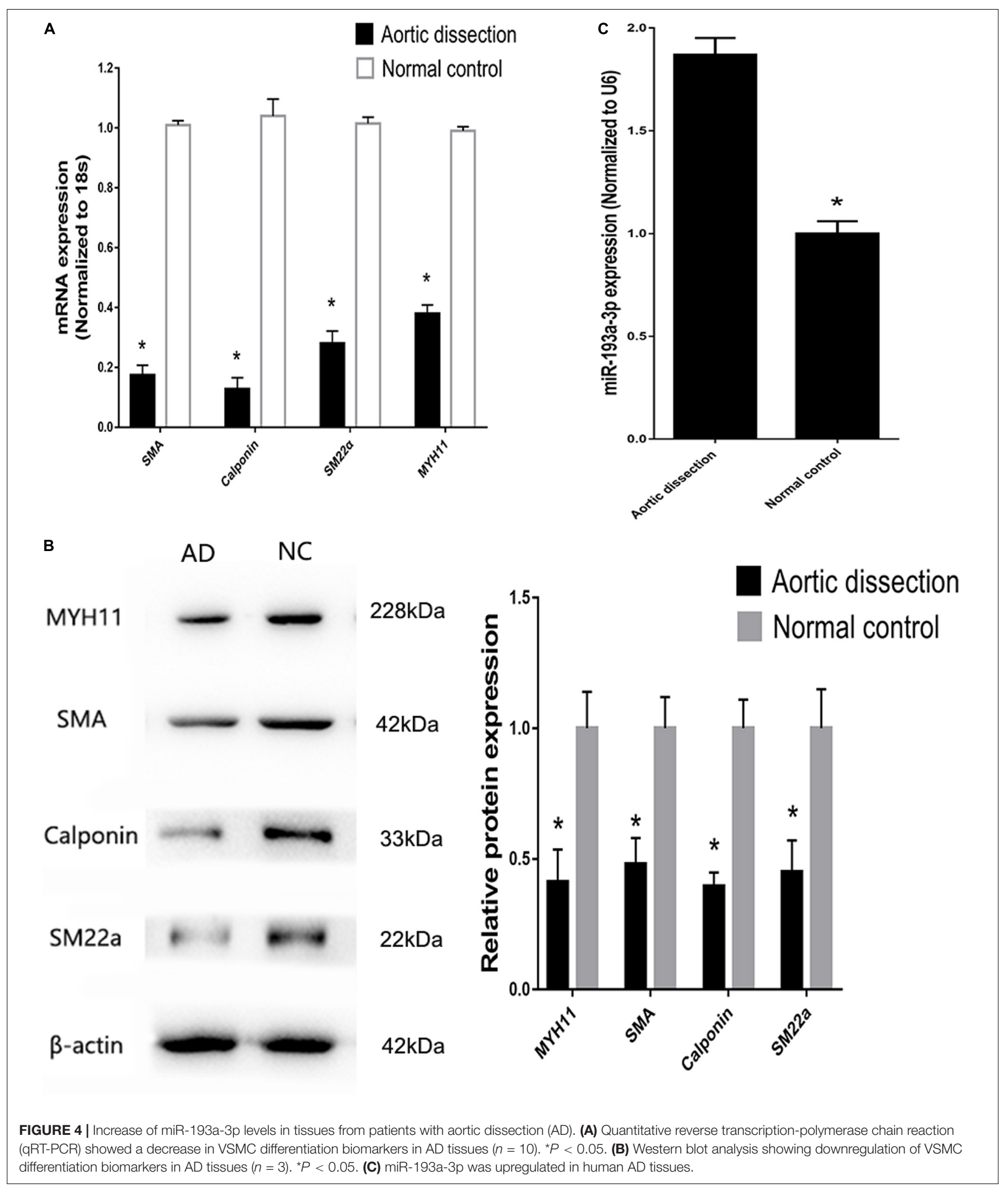

transcription-polymerase chain reaction (qRT-PCR) was used to detect miR-193a-3p expression levels in VSMCs with increasing duration of proliferation $(2,4,6,8,12$, and
$24 \mathrm{~h})$. The qRT-PCR results showed that miR-193a-3p was upregulated in VSMCs with an increase in cell proliferation. As shown in Figure 5, miR-193a-3p was upregulated in 

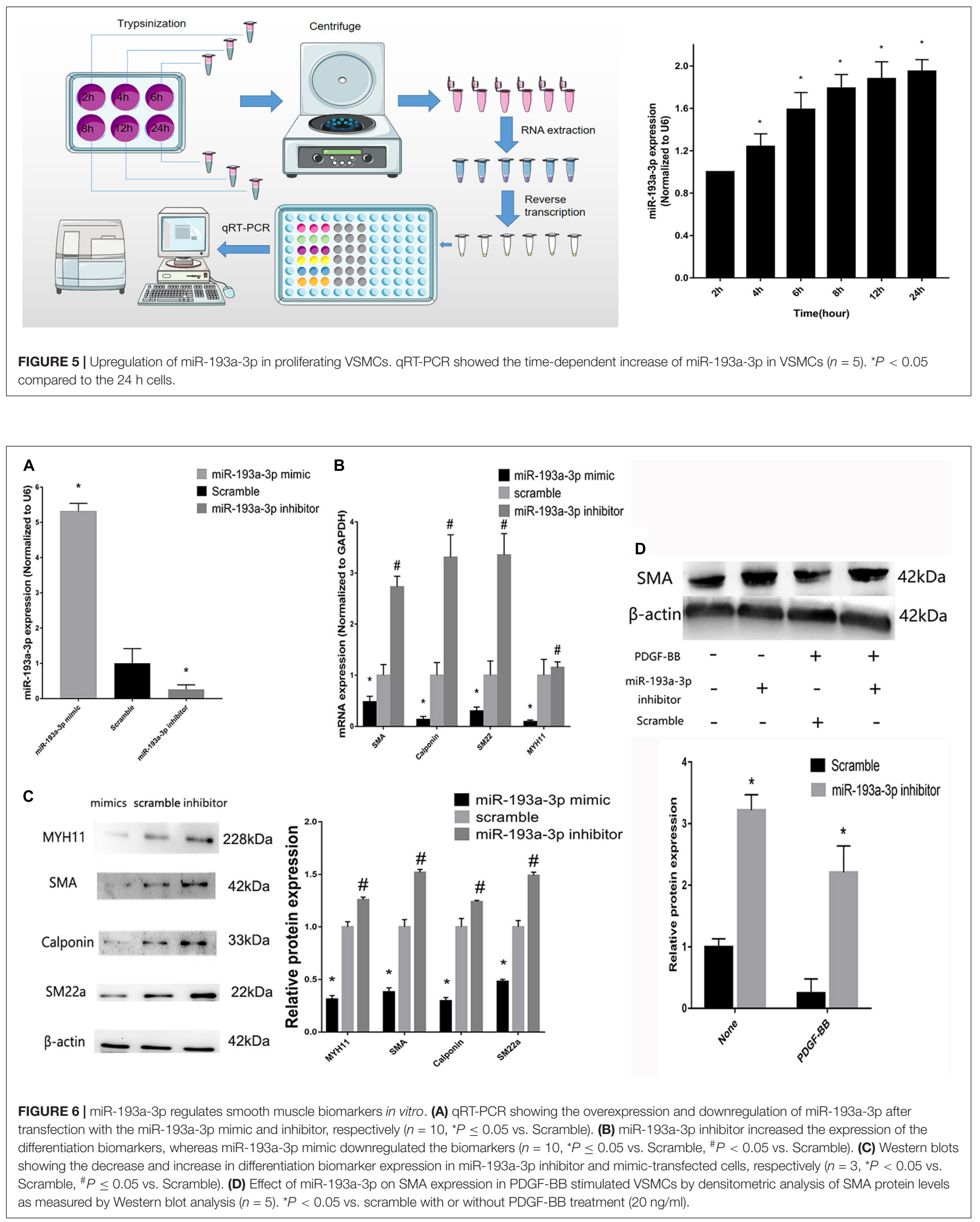
a time-dependent manner together with an increase in VSMC proliferation.

\section{miR-193a-3p Regulates Smooth Muscle Biomarkers in VSMCs}

To investigate the role of miR-193a-3p in human aortic VSMC phenotype switch, we transiently transfected miR-193a-3p mimic and miR-193a-3p inhibitor into human aortic VSMCs. qRT-PCR was used to confirm the transfection efficiency of the miR-193a$3 \mathrm{p}$ mimic, inhibitor, and controls. Figure 6A showed that the miR-193a-3p mimic elevated miR-193a-3p levels, whereas miR193a-3p inhibitor markedly reduced the endogenous levels of miR-193a-3p in VSMCs. Western blotting and qRT-PCR showed the decrease in biomarkers involved in VSMC differentiation, such as SMA, SM22a, calponin, and MYH11 (Figures 6B,C),

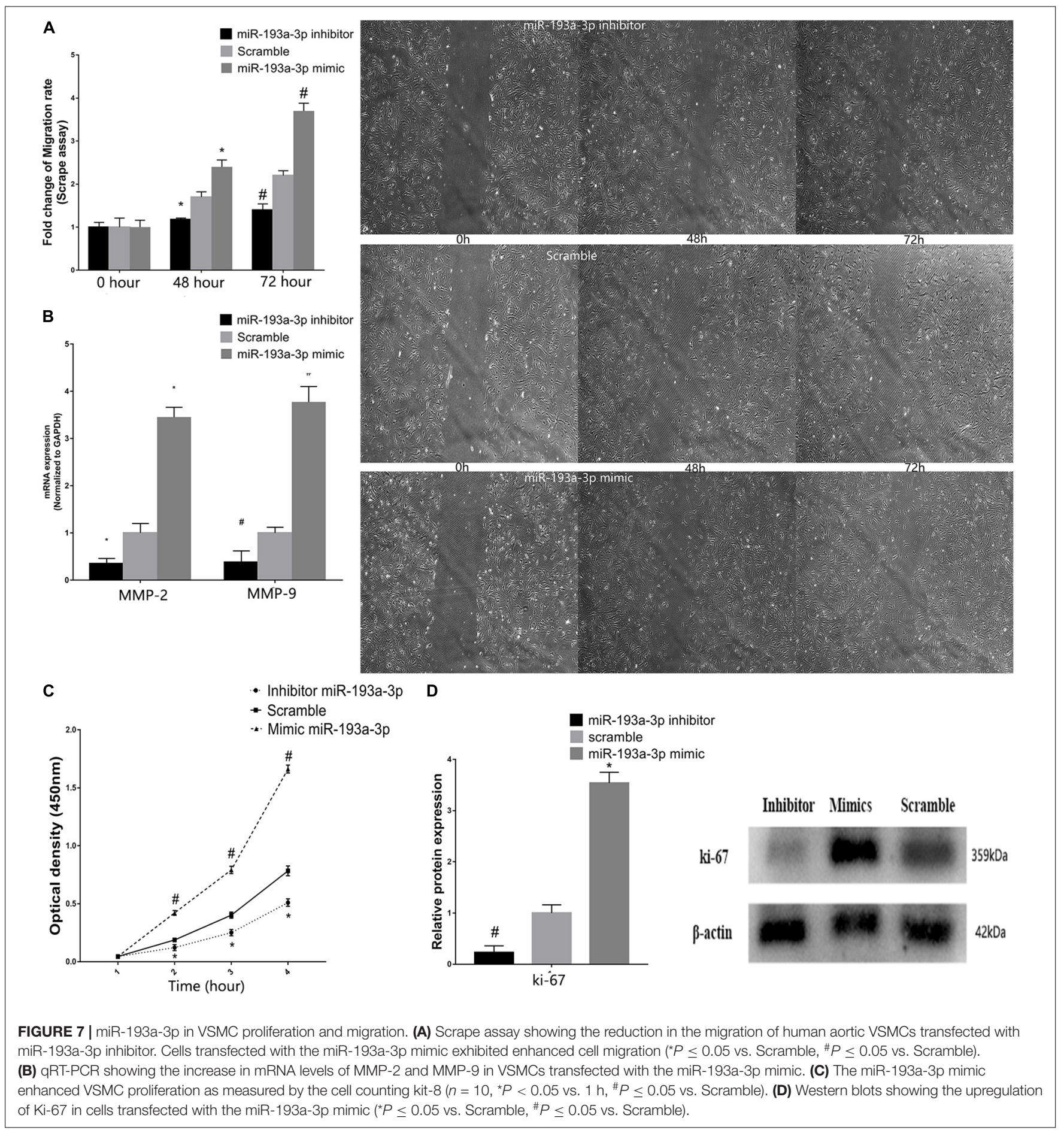


whereas lower levels of miR-193a-3p were associated with upregulation of these biomarkers in human VSMCs. Western blot analysis also demonstrated that the SMA protein levels were dramatically increased after ectopic downregulation of miR193a-3p under both basal and PDGF-BB-stimulated conditions. Thus, miR-193a-3p levels regulated VSMC differentiation.

\section{miR-193a-3p Regulates VSMC Proliferation and Migration}

Vascular smooth muscle cells were transfected with miR-193a$3 \mathrm{p}$ mimic, inhibitor, or control. Subsequently, the scratch assay was used to evaluate the effect of miR-193a-3p on cell migration. Figure 7A showed the marked increase in migration in cells transfected with the miR-193a-3p mimic. In addition, we also detected the migration biomakers such as MMP-2 and MMP-9, which have been implicated in VSMC migration. MMP-2 and MMP-9 RNA levels were also higher in the miR-193a-3p mimic-transfected cells (Figure 7B). These results showed that high expression of miR-193a-3p promoted VSMC migration. As shown in Figure $\mathbf{7 C}$, we assayed the effect of miR-193a-3p on VSMC proliferation using CCK-8. Cell proliferation was recorded at $1,2,3$, and $4 \mathrm{~h}$. Cells overexpressing miR-193a-3p exhibited increased proliferation as compared to the control cells. However, VSMCs depleted of miR-193a-3p showed reduced proliferation. Moreover, we also detected the proliferation biomaker (Ki-67). Western blots showed the upregulation of Ki-67 in miR-193a-3poverexpressing VSMCs, whereas inhibition of miR-193a-3p in VSMCs downregulated Ki-67 (Figure 7D). Taken together, these results indicated that miR-193a-3p was a promoter of VSMC proliferation and migration.

\section{ACTG2 Is a Target of miR-193a-3p}

Figure 8A shows the predicted binding sites for miR-193a-3p within the 3' UTR of ACTG2. We used the dual luciferase reporter assay to detect the effect of miR-193a-3p on the wild-type and mutant $3^{\prime}$ UTR of ACTG2. HEK293T cells overexpressing miR-193a-3p decreased luciferase activity to a greater extent in cells expressing wildtype ACTG2, as compared to that in cells transfected with miR-193a-3p control or expressing mutant

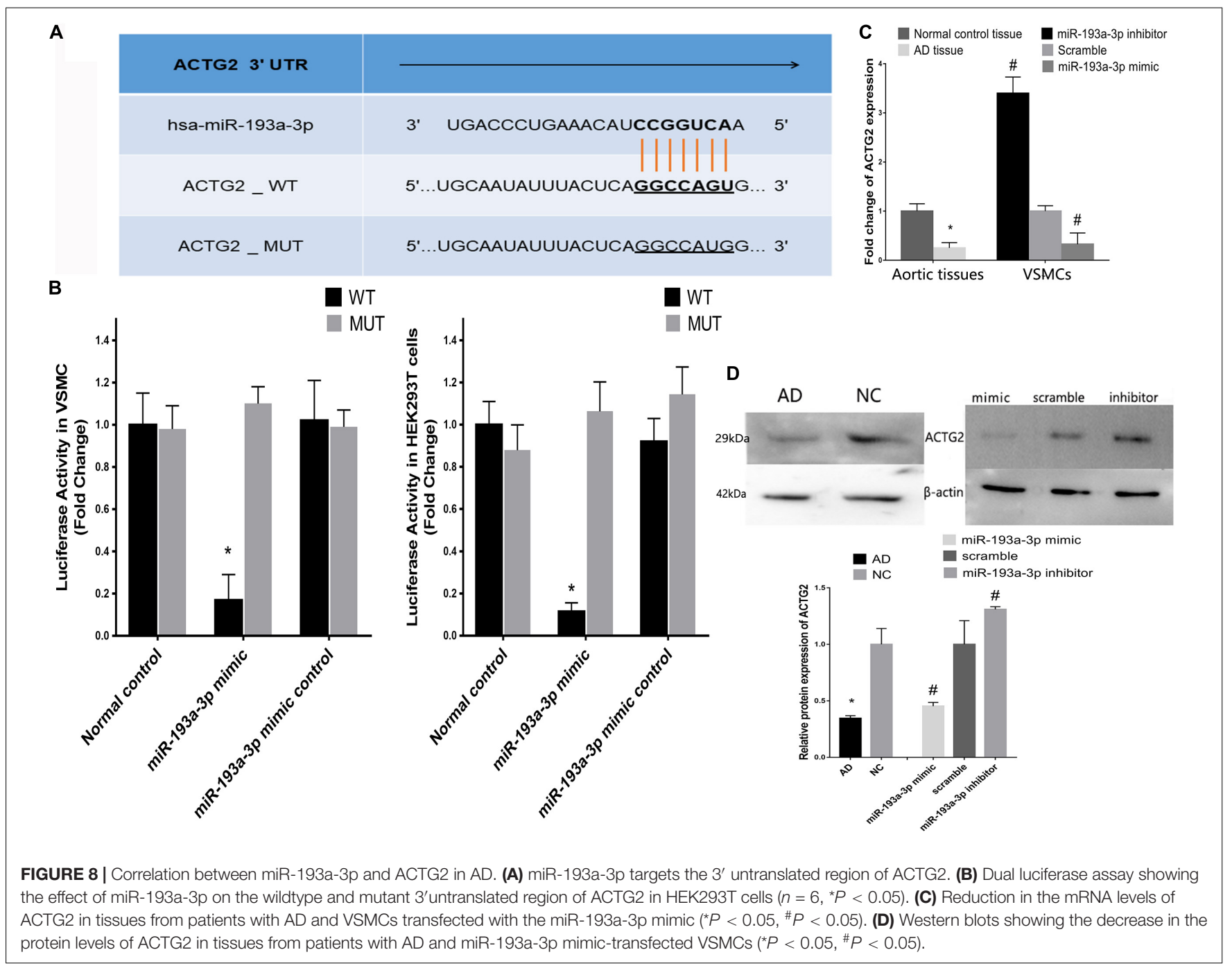


ACTG2 (Figure 8B). Moreover, qRT-PCR revealed that ACTG2 was downregulated in tissues from $\mathrm{AD}$ patients. However, VSMCs transfected with the miR-193a-3p mimic exhibited upregulation of ACTG2 in tissues from healthy individuals and VSMCs depleted of miR-193a-3p (Figures 8C,D).

pcDNA3.1-ACTG2 vector and ACTG2 siRNA were transfected into VSMC for rescue experiments. qRT-PCR was used to verify the transfection efficiency of pcDNA3.1ACTG2 vector and ACTG2 siRNA. pcDNA3.1-ACTG2 vector could elevate the expression of ACTG2 in VSMC, whereas ACTG2 siRNA reduced ACTG2 expression (Figure 9A). miR193a-3p inhibitor + ACTG2 siRNA showed rescue proliferation and migration (Figure 9B) ability compared to that of VSMCs transfected with miR-193a-3p inhibitor only. Phenotypic transition biomarkers involved in VSMC differentiation were lower in miR-193a-3p inhibitor + ACTG2 siRNA than VSMCs transfected with miR-193a-3p inhibitor only (Figures 9C,D).

\section{DISCUSSION}

Vascular smooth muscle cells in aortic media maintain the biological properties of the aortic wall (Rzucidlo et al., 2007; Ren et al., 2017; Chen et al., 2019; Wang et al., 2019b). Proliferation and migration of VSMCs are regulated by phenotypic switching and correlate with the initial stages involved in the development of AD (An et al., 2017a,b; Li et al., 2018; Zhang and Wang, 2019). However, the mechanism(s) of phenotypic switching in VSMCs remain to be understood in detail. In this study, we observed an upregulation of miR-193a-3p in highly proliferative VSMCs and $\mathrm{AD}$ tissues. Up- or downregulating miR-193a-3p resulted in the decrease or increase of the differentiation biomarkers (SMA, MYH11, SM22a, and calponin) in VSMCs, respectively. Therefore, miR-193a-3p stimulates cell proliferation and may be a novel modulator of phenotype switching in VSMCs.

In this study, integrating the miRNA and mRNA profiles and using bioinformatics analyses revealed that the miR-193a3p/ACTG2 axis played an essential role in the pathogenesis of $\mathrm{AD}$. The reason we choose miR-193a-3p/ACTG2 as the target axis is base on the bioinformatic analyses. In addition, PPP1R12B was also predicted as a potential gene in vascular smooth muscle contraction pathway. However, few references reported the interaction between PPP1R12B and vascular smooth muscle contraction pathway as well as AD. miR-193a-3p inhibits the formation, proliferation, and migration of tumor cells in the lung by directly binding to KRAS (Fan et al., 2017). It also functions as a tumor suppressor in colon cancer by interacting with IL17RD (Pekow et al., 2017). However, studies have reported that miR-193a-3p promotes the formation, proliferation, and migration of tumor cells. miR-193a-3p enhances the proliferation and migration of renal carcinoma cells by directly targeting PTEN (Liu et al., 2017). Furthermore, it promotes the development of

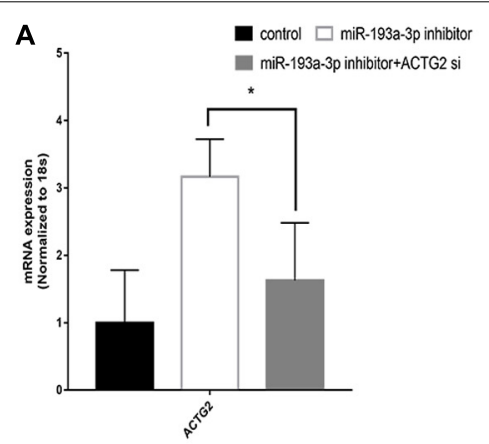

C

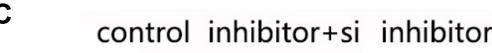

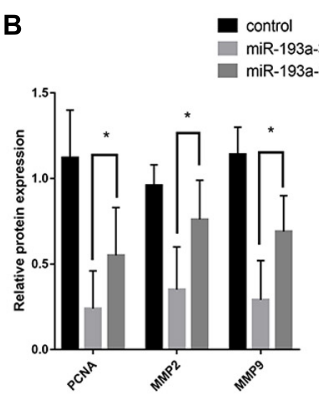

miR-193a-3p inhibitor

miR-193a-3p inhibitor+ACTG2 si

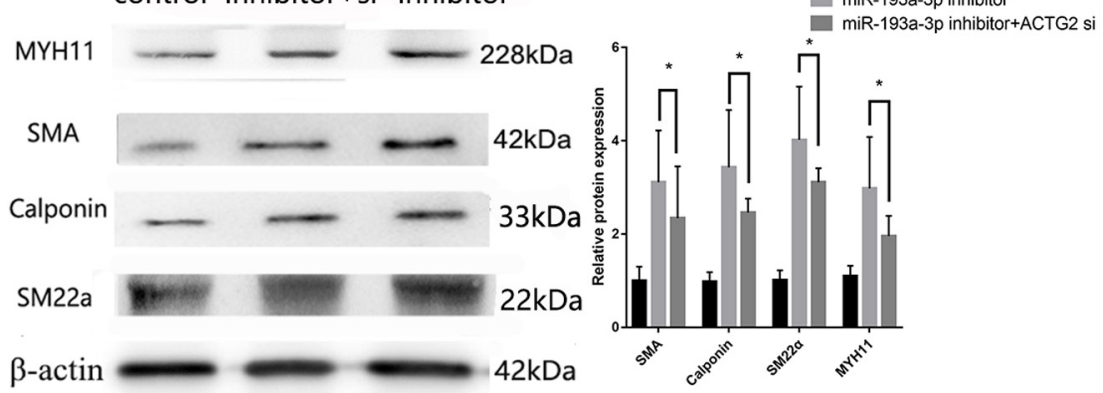

D

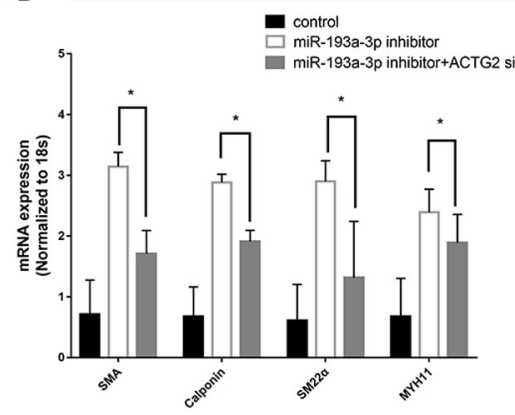

FIGURE 9 | Rescue experiments of ACTG2. (A) qRT-PCR presented over/downregulation of ACTG2 after transfection with ACTG2 vector and siRNA, respectively $\left(n=3,{ }^{*} P \leq 0.05\right.$ vs. control). (B) Western blot showed remedy PCNA, MMP2, and MMP9 after transfection with miR-193a-3p inhibitor + ACTG2 siRNA than transfection with miR-193a-3p inhibitor only ( $n=10,{ }^{*}$ vs. miR-193a-3p inhibitor). (C) Western blot analysis showed that low expression of VSMCs differentiation biomarkers in cells transfected with miR-193a-3p inhibitor + ACTG2 siRNA comparing to transfection with miR-193a-3p inhibitor only $(n=3$, * vs. miR-193a-3p inhibitor). (D) qRT-PCR presented remedy expression of VSMC differentiation biomarkers. 
bladder cancer by targeting HOXC9 (Faugeroux et al., 2015). However, the role of miR-193a-3p on cardiovascular disease, especially $\mathrm{AD}$, has not been studied. Therefore, the identification of miR-193a-3p as a modifier of VSMC phenotype and proliferation will increase our understanding of the pathogenesis of heart disease.

We observed that increased expression of miR-193a-3p (in $\mathrm{AD}$ tissues) correlated with the reduced expression of differentiation biomarkers in VSMCs. Furthermore, miR-193a-3p was overexpressed in proliferating human aortic VSMCs that had been proliferating for longer durations. CCK-8 and scrape assays showed that the miR-193a-3p mimic and inhibitor increased and suppressed the proliferation and migration of human VSMCs, respectively. These results help strengthen the inference that miR193a-3p may be a modulator of phenotypic switching in VSMCs.

MicroRNAs regulate translation by binding to the $3^{\prime}$ UTRs of target mRNAs (Liu et al., 2019). Using various molecular biology techniques and bioinformatics analyses, we have demonstrated that ACTG2 is a target of miR-193a-3p. Overexpression of miR193a-3p resulted in the downregulation of ACTG2 in transfected VSMCs. These VSMCs exhibited enhanced proliferation and reduced expression of the differentiation biomarkers SMA, MYH11, SM22a, and calponin.In addition, we found the converse regulation between miR-193a-3p and the differentiation markers. So targetscan bioinformatic analyses toold was used to predict binding sites of miR-193a-3p. The results showed no binding sites between miR-193a-3p and SMA, MYH11, SM22a, and calponin. Therefore, we concluded that miR-193a-3p could not directly regulate these differentiation markers.

However, this study also contains severel limitations. All in vitro experiments were performed in VSMCs, the different expression of ACTG2 and miR-193a-3p in endothelial remained unclear. In addition, two gene profiles were used in our study, which might cause bias. More sequencing results need to be used for co-expression analysis. Finally, the potential roles of PPP1R12B in VSMCs phenotypic transition need further verification experiments.

In summary, our study revealed that miR-193a-3p was increased in ascending aortic tissues from AD patients. Moreover,

\section{REFERENCES}

Alexander, M. R., and Owens, G. K. (2012). Epigenetic control of smooth muscle cell differentiation and phenotypic switching in vascular development and disease. Annu. Rev. Physiol. 74, 13-40. doi: 10.1146/annurev-physiol-012110142315

An, Z., Liu, Y., Song, Z. G., Tang, H., Yuan, Y., Xu, Z.-Y., et al. (2017a). Mechanisms of aortic dissection smooth muscle cell phenotype switch. J. Thorac. Cardiovasc. Surg. 154, 1511-1521. doi: 10.1016/j.jtcvs.2017. 05.066

An, Z., Qiao, F., Lu, Q., Ma, Y., Liu, Y., Lu, F., et al. (2017b). Interleukin-6 downregulated vascular smooth muscle cell contractile proteins via ATG4Bmediated autophagy in thoracic aortic dissection. Heart Vessels 32, 1523-1535. doi: 10.1007/s00380-017-1054-8

Chen, D., Zang, Y. H., Qiu, Y., Zhang, F., Chen, A.-D., Wang, J.-J., et al. (2019). BCL6 attenuates proliferation and Oxidative stress of vascular smooth Muscle Cells in Hypertension. Oxid. Med. Cell Longev. 2019:5018410.

Elsayed, R. S., Cohen, R. G., Fleischman, F., and Bowdish, M. E. (2017). Acute Type A Aortic dissection. Cardiol. Clin. 35, 331-345.
miR-193a-3p targeted the $3^{\prime}$ UTR of ACTG2 and may be a novel regulator of phenotypic switching, proliferation, and migration in VSMCs. Thus, the miR-193a-3p/ACTG2 axis may provide mechanistic insight into the pathogenesis of $\mathrm{AD}$ and serve as a promising diagnostic biomarker and therapeutic target for $\mathrm{AD}$.

\section{DATA AVAILABILITY STATEMENT}

The datasets presented in this study can be found in online repositories. The names of the repository/repositories and accession number(s) can be found below: GSE98770 and GSE52093.

\section{ETHICS STATEMENT}

This study was conducted in accordance with the Declaration of Helsinki and was approved by the Ethical Committee of Jilin University. All patients agreed to participate this program and provided written informed consent.

\section{AUTHOR CONTRIBUTIONS}

WW designed and supervised the study. TW, KL, HP, YW, and $\mathrm{BL}$ performed the analysis work. ZZ and DL contributed to the data analysis. WW and KL organized, designed, and wrote the manuscript. All authors reviewed the final manuscript.

\section{FUNDING}

This work was supported by the project of National Natural Science Foundation of China, China (Grant no. 81970399), the project of special health project of Jilin Provincial Department of Finance, China (Grant no. 20180101), the project of Jilin Key Laboratory Construction Project, China (Grant no. 20190901008JC).

Fan, Q., Hu, X., and Zhang, H. (2017). MiR-193a-3p is an important tumour suppressor in lung cancer and directly targets KRAS. Cell Physiol. Biochem. 44, 1311-1324. doi: 10.1159/000485491

Faugeroux, J., Lv, L., Li, Y., Deng, H., Zhang, C., Pu, Y., et al. (2015). MiR-193a-3p promotes the multi-chemoresistance of bladder cancer by targeting the HOXC9 gene. Cancer Lett. 357, 105-113. doi: 10.1016/j.canlet.2014.11.002

Gomez, D., and Owens, G. K. (2012). Smooth muscle cell phenotypic switching in atherosclerosis. Cardiovasc. Res, 95, 156-164. doi: 10.1093/cvr/cvs115

Li, R., Yi, X., Wei, X., Huo, B., Guo, X., Cheng, C., et al. (2018). EZH2 inhibits autophagic cell death of aortic vascular smooth muscle cells to affect aortic dissection. Cell Death Dis. 9:180.

Li, X. L., de Molfetta, G. A., and Ramão, A. (2019). miR-450a acts as a tumor suppressor in ovarian cancer by regulating energy metabolism. Cancer Res. 79, 3294-3305. doi: 10.1158/0008-5472.can-19-0490

Liu, L., Li, Y., Liu, S., Duan, Q., Chen, L., Wu, T., et al. (2017). Downregulation of miR-193a-3p inhibits cell growth and migration in renal cell carcinoma by targeting PTEN. Tumour. Biol. 39:1010428317711951.

Liu, Y., Chen, G., Liu, H., Li, Z., Yang, Q., Gu, X., et al. (2019). Integrated bioinformatics analysis of miRNA expression in Ewing sarcoma and potential 
regulatory effects of miR-21 via targeting ALCAM/CD166. Artif. Cells Nanomed.Biotechnol. 47, 2114-2122. doi: 10.1080/21691401.2019.1620760

Pekow, J., Meckel, K., Dougherty, U., Huang, Y., Chen, X., Almoghrabi, A., et al. (2017). miR-193a-3p is a Key tumor suppressor in ulcerative colitis-associated colon cancer and promotes carcinogenesis through upregulation of IL17RD. Clin. Cancer Res. 3, 5281-5291. doi: 10.1158/1078-0432.ccr-17-0171

Ren, X. S., Tong, Y., Ling, L., Chen, D., Sun, H.-J., Zhou, H., et al. (2017). NLRP3 gene deletion attenuates angiotensin II-induced phenotypic transformation of vascular smooth Muscle Cells and vascular remodeling. Cell Physiol. Biochem. 44, 2269-2280. doi: 10.1159/000486061

Rzucidlo, E. M., Martin, K. A., and Powell, R. J. (2007). Regulation of vascular smooth muscle cell differentiation. J. Vasc. Surg. 45, A25-A32.

Silaschi, M., Byrne, J., and Wendler, O. (2017). Aortic dissection: medical, interventional and surgical management. Heart 103, 78-87. doi: 10.1136/ heartjnl-2015-308284

Tang, Y., Yu, S., Liu, Y., Zhang, J., Han, L., Xu, Z., et al. (2017). MicroRNA-124 controls human vascular smooth muscle cell phenotypic switch via Sp1. Am. J. Physiol. Heart Circ. Physiol. 313, 641-649.

Wang, W., Liu, Q., Wang, Y., Piao, H., Li, B., Zhu, Z., et al. (2019a). Verification of hub genes in the expression profile of aortic dissection. PLoS One 14:e0224922. doi: 10.1371/journal.pone.0224922

Wang, W., Wang, T., Wang, Y., Piao, H., Li, B., Zhu, Z., et al. (2019b). Integration of gene expression profile data to verify hub genes of patients with stanford A Aortic dissection. Biomed. Res. Int, 2019:3629751.
Wei, X., Sun, Y., Wu, Y., Zhu, J., Gao, B., Yan, H., et al. (2017). Downregulation of Talin-1 expression associates with increased proliferation and migration of vascular smooth muscle cells in aortic dissection. BMC Cardiovasc. Disord. 17:162. doi: 10.1186/s12872-0170588-0

Zhang, M., and Wang, Z. (2019). Downregulation of miR143/145 gene cluster expression promotes the aortic media degeneration process via the TGF- $\beta 1$ signaling pathway. Am. J. Transl. Res. 11, 370-378.

Zhang, P., Hou, S., Chen, J., Zhang, J., Lin, F., Ju, R., et al. (2016) Smad4 Deficiency in Smooth Muscle Cells Initiates the Formation of Aortic Aneurysm. Circ. Res. 118, 388-399. doi: 10.1161/circresaha.115.30 8040

Conflict of Interest: The authors declare that the research was conducted in the absence of any commercial or financial relationships that could be construed as a potential conflict of interest.

Copyright (c) 2021 Wang, Wang, Piao, Li, Zhu, Li, Wang and Liu. This is an openaccess article distributed under the terms of the Creative Commons Attribution License (CC BY). The use, distribution or reproduction in other forums is permitted, provided the original author(s) and the copyright owner(s) are credited and that the original publication in this journal is cited, in accordance with accepted academic practice. No use, distribution or reproduction is permitted which does not comply with these terms. 